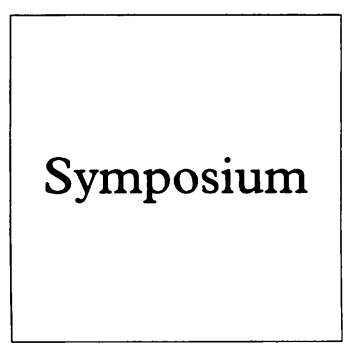

\title{
Is Europe ready for STD screening?
}

Per-Anders Mårdh

Is screening cost beneficial as a strategy to combat the epidemic of genital chlamydial infections and some other sexually transmitted diseases (STDs)? What are the medical, human, and social benefits and costs of screening? Finally, are we ready to start a programme of more general screening of STD agents within the populations of Europe?

There are a number of reasons to screen for Chlamydia trachomatis. At least half of infected people are carriers-that is, they either do not experience sufficient symptoms to feel motivated to consult, or they simply have no symptoms at all. Screening and identifying such cases allows detection of additional chlamydia positive cases through partner notification. This is highly rewarding as more than half of all partners of a chlamydia infected index case are also likely to be infected. ${ }^{1}$ This is true even when less sensitive detection methods, such as ELISA (enzyme linked immunosorbent assay) are used. Another important reason to screen is that adverse immunological reactions may be induced by chlamydial infections such as production of chlamydial heat shock protein (HSP) antibodies, which cross react with human HSP. Such adverse immune reactions might not be abolished by eradicating the organism by antibiotic therapy; thus, breaking the transmission chains by detection and treatment of carriers is important.

The overall effect of reducing the number of chlamydial infections can be demonstrated by analysis of the situation in Sweden (a country where the notification of infections by Chlamydia trachomatis has been obligatory since 1982), where the number of reported cases has dropped from approximately 40000 cases in 1987 to around 15000 cases in $1994 .^{2}$ The introduction of more sensitive diagnostic techniques, PCR (polymerase chain reaction) and LCR (ligase chain reaction), in the most recent years resulted in only a marginal increase in reported chlamydial infections. Furthermore, there has recently been increased screening for $C$ trachomatis among Swedish men. Along with this drop in prevalence there has been a corresponding reduction in the number of cases of pelvic inflammatory disease (PID) ${ }^{3}$ and of ectopic pregnancies, ${ }^{4}$ two of the known complications of genital chlamydial infections. A drop in prevalence has also been seen in parts of the USA which have instigated screening programmes for genital chlamydial infection. ${ }^{5}$ Whether or not there has been or will be a similar drop in the number of cases of infertility in these places, is, however, not yet known.

Among the obstacles to the introduction of wider STD screening in Europe is, of course, the current critical situation of health econom- ics, particularly in eastern Europe. The message that a society may gain and not lose money in the long run ${ }^{67}$-that is, in a perspective of a decade, by screening for, for example, $C$ trachomatis, may not be immediately attractive to those responsible for the management of healthcare budgets, particularly budgets that rely on government money. New expenditure is seldom sanctioned for benefits that are discounted over such a time perspective. In this context it is necessary to realise that the mean time lag that passes after a primary tubal chlamydial infection and before a woman attends because of PID or ectopic pregnancy is approximately $7-8$ years. These two conditions are costly to society. Abdominal pain in PID is likely to result in an increased consumption of health care and pain relieving drugs etc, adding costs to the health budget. Many social costs due to STDs, their complications, and sequelae have not generally been considered in cost-benefit calculations; costs which may exceed several fold the costs of detection and treatment of the original condition.

It has been calculated that it is cost beneficial to screen for $C$ trachomatis when the prevalence of genital chlamydial infection is approximately $6 \%^{6}$ and when treating all detected cases with $1 \mathrm{~g}$ of azithromycin as a single dose (assuming a non-existent compliance problem if the drug is given at a clinic). In another $\mathrm{study}^{8}$, which used different criteria for diagnosing PID, included an estimate of the number of silent PIDs, and used other methods for detecting chlamydia infection, a prevalence of $3 \%$ was found to be the cut off for screening shown to be cost beneficial.

In 24 family planning clinics in 14 European countries, we found the prevalence of $C$ o trachomatis infections in 3000 women aged $15 \mathrm{~N}$ to 45 years, who considered themselves to be gynaecologically healthy, to range from $0.0 \%$ 음 to $7 \cdot 6 \% .9^{9}$ The study made use of a PCR 0 method for testing urine samples. In five of these countries, the prevalences were above ? $3 \%$. Selective screening is likely to increase the cost-benefit rates in populations with overall $\mathbb{D}$ prevalences below the threshold for gain. Thus, screening of cohorts of women with known risk factors for infection or symptomatic cases-for example, women complaining of vaginal discharge, is likely to identify even higher prevalences than in asymptomatic cohorts. Furthermore, if partner notification is undertaken among the sexual partner(s) of individuals found positive at screening and also of the partner's partner(s), such case detection is likely to increase the cost-benefit further.

Which methods should be used for screening? The method used must be suitable for its 
purpose-that is, the right method for the right type of sample for a given person or population being examined. In mass screening it is important that the method will allow non-invasive sampling. Nucleic acid based methods must be recommended if economy allows. ${ }^{6710}$ LCR or PCR can be used for urethral/cervical samples, for voided urine samples, ${ }^{11} 12$ and also for vaginal introital specimens ${ }^{13}$; the latter with the same or ever higher sensitivity than when analysing any other type of sample by the same methodology.

The advantage of using a PCR or LCR method is that these methods allow simultaneous detection of more than one STD agentfor example, diagnosis of a genital chlamydial infection and gonorrhoea by testing the same urine sample. In the future it seems likely that a whole battery of primers of sexually transmittable agents may be used, which will increase the motivation to introduce STD screening programmes. Screening in certain age groups for oncogenic types of human papillomavirus (HPV) are among top candidates for such an initiative. ${ }^{14}$ In eastern Europe tests for syphilis are becoming another top priority for STD screening programmes, although this will require blood to be drawn for serological tests. Such tests for HPV antibodies may also be informative. ${ }^{15}$

The use of PCR and LCR has increased the percentage of infected people detected, compared with previously used methods, such as DIF (direct immunofluorescence) and ELISA. The exact increase in sensitivity achieved by introducing these new methods is speculative, but for $C$ trachomatis has ranged from $10 \%$ to at least $50 \%$ depending on sample type and test population studied. Another factor is the performance quality of earlier methods used, such as that of tissue cell culture for detection of chlamydiae. When testing cases of chronic chlamydial infections in which the chlamydia antigen load may be very low, the use of DNA/RNA based methods is even more essential. Nucleic amplification methods are not without their problems, however. ${ }^{16}{ }^{17}$

A low antigen load may be more common among women in their thirties with chronic long standing chlamydial infections than among female teenagers with acute primary infections. We found little variation by age in the prevalence of chlamydia positive cases in the European screening study of family planning attendees aged 18-45 years referred to above, in which we used a PCR test. ${ }^{9}$ Our study indicated that focusing only on older teenagers and women in their twenties in screening programmes for $C$ trachomatis is not likely to be an optimal approach if one aims to obtain a maximum health gain-that is, a maximum number of case detected, treated, and thus the prevention of the spread of the infection to non-infected individuals. However, the cost-benefit of such extended screening programmes remains to be determined in attempts to reduce the reservoir of the organism in the general European female population.

Which method one should use for screening for genital chlamydial infection also depends on the type of sample available-for example, a voided urine sample or a cervical swab and what techniques are available in the local laboratory as well as the prevailing conditions for storage and transport of samples. One essential question for sampling is that of whether the specimen to be tested is to be collected at a clinic with a gynaecological examination chair available. So far, almost all epidemiological data on genital chlamydial infections in females have been restricted to populations with this test prerequisite. Also, the extent to which existing laboratory equipment can be adapted to new methods and the experience of the laboratory staff can be essential in determining what test to recommend. Paradoxically, such advanced technologies, such as PCR and LCR, may be less operator dependent than tissue cell cultures and DIF in laboratories where the staff has less experience of chlamydia diagnostic work. This is particularly so if one can make use of automated laboratory equipment to perform such nucleic acid based tests. Handling tissue cell cultures and reading DIF tests of urine sediments to detect elementary bodies of $C$ trachomatis, requires highly trained personnel. Furthermore, knowledge of the approximate prevalence in groups to be tested is essential if an ELISA is to be used; ELISA tests may have a positive predictive value as low as $50 \%$ in low prevalence populations-for example, in screening situation of asymptomatic individuals. Thus, communication between the laboratory and clinic is essential to find out which tests should be used for which cohorts. In this context it should be remembered that in eastern Europe the only tests available today are generally ELISA, DIF tests, and, in some places, culture. Economic stringencies in these countries do not as yet generally allow nucleic acid tests to be used.

What about the type of sample to be tested? For example, urine is not an appropriate sample for chlamydia culture because of inhibitory factors in the urine. ELISA also works poorly for this type of sample when compared with nucleic acid based tests. Urine requires access at the clinic to some, albeit simple, laboratory equipment (for example, a table centrifuge, a refrigerator, or a deep freeze) if the sample has to be stored for some days before being sent to the laboratory. In fact, we found freezing and thawing of urine twice increased the detection rate of $C$ trachomatis by PCR (Bassiri et al, unpublished data).

Use of antibiotics (for any reason) is common and may interfere with culture for detection of $C$ trachomatis. When antibiotics active against $C$ trachomatis have been used near to the time of sampling, the treated infection might still be detectable by PCR. That is true even if the infection has been microbiologically cured. Such information may be useful as it can support decisions on partner notification.

Are there at present any obstacles, apart from technical ones, to the introduction of screening for $C$ trachomatis and $N$ gonorrhoeae in Europe? One obvious obstacle, at least in the eastern European countries with poor economies, is that even people who have con- 
sulted and who are complaining of genital symptoms cannot afford to be tested. Thus, in several of these countries any necessary laboratory test has to be paid for by the patient $\mathrm{him} / \mathrm{herself}$. This may also be the case in many state owned hospitals.

It might be difficult to convince holders of budgets for health services that money should be allocated to test people who appear healthy (the carriers) to prevent disease in other people (partners) and to prevent sequelae that might not incur healthcare costs until almost a decade after the screening test is performed.

Also, in countries with comparatively good economic resources there are still a number of obstacles to the introduction of more generalised screening programmes. One such obstacle is the lack of knowledge among the medical profession (that is, those who should promote screening activities) of the consequences of chlamydial infections.

An awareness of these consequences is required by physicians and workers in many disciplines since patients affected by STD infections such as chlamydiae may turn up in a variety of clinics. The range of services visited underscores the extent of the problems caused by these infections. For example, chlamydia infected patients may be seen in ophthalmological, surgical, gynaecological, paediatric, rheumatological, venereological, infectious disease, and internal medicine clinics, as well as by general practitioners.

The acceptance not only by the medical community of the necessity to screen for STD agents but also by the general population is, of course, essential if such screening programmes are to be introduced successfully. In this context, it should be mentioned that when we performed screening for $C$ trachomatis in the 3000 European women attending family planning clinics referred to above, ${ }^{10}$ there was no resistance to our proposal to participate from anyone approached. Furthermore, none of the 900 Lithuanian women whom we recently screened (Domeika et al, unpublished data) refused to perform vaginal introital self sampling.

The decision makers of whether or not screening should be introduced on a large scale in individual European countries can vary from the physician in charge of a hospital department or a laboratory, to a hospital director, or a provincial or even government representative. In some eastern European countries, import of goods, like test kits (to be used by society health providers), may require authorisation by the national board of health, which may seem strange to many western Europeans.

All screening activities may result in new costs as a result of accidental detection of other diseases at follow up. However, such case detection made at an early stage of the disease may either add to the pay off for the screening programme or produce new costs. To combine STD screening with screening for other preventable conditions, such as cervical cancer, should be considered. Similarly, combining screening for STDs with contraceptive advice, counselling about selected health risks (for example, smoking) or general health risks (overweight and absence of physical exercise) may help to reduce healthcare costs on a long term basis along with cost reductions achieved by the STD screening programme.

The possible use of home testing for STDs, in a similar way to home pregnancy testing (tests for which can be bought over the counter, even in tobacco stores in some countries) is becoming closer to reality. Such tests have already emerged on the market for the detection of genital $C$ trachomatis infections. The disadvantage of a home test situation where urine, saliva, and swabs of other clinical material are collected, is that sample identity control is missed, as well as any impact on sampling quality, storage, and transport conditions. We (Hellberg and Mårdh, unpublished data) have developed a client based questionnaire where individuals answer general questions which will discriminate between STD infected and non-infected people and where the risk of being an STD carrier is given as a percentage for different age groups. The spread of such a scoring system might encourage more individuals who have reason to attend an STD clinic to do so.

1 Thelin $\mathrm{K}$, Wennström A-M, Mårdh P-A. Contact tracing in patients with genital chlamydial infection. $B r \mathcal{F}$ Vener Dis patients with gen

2 Smittskydd 1996 vol 2, (Swedish) Stockholm: Smittskyddsinstitutet.

3 Kamwendo F, Forslin L, Bodin L. Decreasing incidences of gonorrhoea- and chlamydia-associated accute pelvic inflammatory disease-a 25-year study from an urban area of central Sweden. Sex Transm Dis 1996;23:384-91.

4 Thorburn J. Utomkvedshavandeskap. Epidemin tycks vara över. Läkartidningen 1995;26:167-70 (Swedish).

5 Scholes D, Stergachis A, Heidrich FE, Andrilla H, Holmes $\mathrm{KK}$, Stamm WE. Prevention of pelvic inflammatory disease by screening for cervical chlamydial infection. $N$ Engl 7 Med 1996;334:1362-6.

6 Genç M, Ruusuvaara L, Mårdh P-A. An economic evaluation of screening for Chlamydia trachomatis in adolescent males. $\mathcal{F} A M A$ 1993;17:2057-64.

7 Genç M, Mårdh P-A. A cost-effectiveness analysis of screening and treatment for Chlamydia trachomatis infection in asymptomatic women. Ann Intern Med 1996;124: 1-7.

8 Paavonen J, Puolakkainen $M$, Paukku $M$, Sintonen $\mathrm{H}$. Cost-benefit analysis of screening for cervical chlamydial infection in low prevalence population. Proceedings Third Meeting of the European Society of Chlamydia Research, Vienna, Austria, September 1996, 11-4.

9 Mårdh P-A, Genç M. Is Europe ready for screening for genital chlamydial infections? Third European Chlamydia Meeting, 11-14 September 1996. University of Vienna, Vienna, 381-4.

10 Buimer M, Van Doornum GJ, Prince M, Henquet C, Cutinhu RA, Plier P, et al. Detection of Chlamydia trachomatis infection in urine samples from men and women by ligase chain reaction. $\mathcal{F}$ Clin Microbiol 1995;33:2042-7.

11 Chernesky MA, Jan D, Lee H, Burczak JD, Hu H, Sellors J, et al. Diagnosis of Chlamydia trachomatis infections in men and women by testing first void urine by ligase chain reaction. $\mathcal{F}$ Clin Microbiol 1994;32:2682-5.

12 Bassiri M, Hu HY, Domeika M, Burczak J, Svensson L-O, Lee $\mathrm{HH}$, et al. Detection of Chlamydia trachomatis in urine specimens from women by ligase chain reaction (LCR). $\mathcal{F}$ Clin Microbiol 1995;33:898-900.

13 Witkin SS, Inglis SR, Polaneczky M. Detection of Chlamydia trachomatis and Trichomonas vaginalis by polymerase chain reaction in introital specimens from pregnant women. Am $\mathcal{f}$ Obstet Gynecol 1996;175:165-7.

14 Cuzick J, Szarewski A, Terry G, Ho L, Hanby A, Maddox $\mathrm{P}$, et al. Human papillomavirus testing in primary cervical $P$, et al. Human papillomavirus testing in primary cervical

screening. Lan

, Koskela $P$, Lehtinen $M$, et al. Seropositivities to human papillo- ํㅡㅁ mavirus types 16,18 or 33 capsids and to Chlamydia trachomatis are markers of sexual behaviour. $\mathcal{F}$ Infect $D i$ 1996;173:1394-8.

16 Schachter J, Stamm WE, Chernesky MA, Hook EW, Jones RB, Judson FN, et al. Nonculture tests for genital tract chlamydial infection. What does the package insert mean, and will it mean the same tomorrow? Sex Transm Dis 1992;19:243-4.

17 Jensen IP, Thorsen P, Møller BR. Sensitivity of ligase chain reaction assay of urine from pregnant women for Chlamydia trachomatis. Lancet 1997;349:329-30. ,

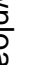

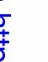

